\title{
ON THE NOETHERIAN-LIKE RINGS OF E. G. EVANS
}

\author{
WILLIAM HEINZER AND JACK OHM ${ }^{1}$
}

\begin{abstract}
ABSTRACr. It is shown that if a commutative ring with identity $R$ is nonnoetherian, then the polynomial ring in one indeterminate over $R$ has an ideal with infinitely many maximal prime divisors (in the sense of Nagata).
\end{abstract}

Let $R$ denote a commutative ring with 1 , and for any ideal $A$ of $R$, let $\mathscr{Z}(A)=\{r \in R \mid$ there exists $s \in R \backslash A$ such that $r s \in A\}$. (By "ideal" we shall always mean ideal $\neq R$. The notation $R \backslash A$ denotes the set-complement of $A$ in $R$.) $\mathscr{Z}(A)$ is merely the set of zero-divisors on the $R$-module $R / A$ and is always a union of prime ideals of $R$. Evans [1] calls $R$ a ZDring (zero-divisor ring) if for any ideal $A$ of $R, \mathscr{Z}(A)$ is a union of finitely many prime ideals. We shall prove here the following:

THEOREM. $R$ is noetherian if (and only if) the polynomial ring in one indeterminate $R[X]$ is a $Z D$-ring.

Evans has proved in [1] the 2 indeterminate analogue of this theorem (which follows from the theorem) and the special case of the theorem for $R$ containing an infinite field.

A prime ideal $P$ of $R$ such that $P$ is maximal with respect to the property of being contained in $\mathscr{Z}(A)$ is called a maximal $\mathrm{N}$-prime (for Nagataprime) of $A$. Note that such a prime contains $A$ and that $\mathscr{Z}(A)$ is the union of the maximal N-primes of $A$. (See [2] and [4] for a perspective on the associated primes of an ideal.)

Proof of Theorem. Suppose $R$ is not noetherian. Then there exists a strictly ascending chain $(0)<\left(a_{1}\right)<\left(a_{1}, a_{2}\right)<\cdots<\left(a_{1}, \cdots, a_{n}\right)<\cdots$ of ideals of $R$. Let $f_{0}=X, f_{1}=1+X, \cdots, f_{i}=1+f_{0} f_{1} \cdots f_{i-1}, \cdots$. We wish to show that the ideal $A=\left(a_{1} f_{1}, a_{2} f_{1} f_{2}, \cdots, a_{n} f_{1} \cdots f_{n}, \cdots\right)$ in $R[X]$ has an infinite number of maximal $\mathrm{N}$-primes and hence has the property that $\mathscr{Z}(A)$ is not a finite union of prime ideals. We show first that each $f_{i} \in \mathscr{Z}(A)$. Since $A \subset\left(f_{1}\right)$ and $f_{1}$ is a monic polynomial of positive degree in $R[X]$, it follows that $A \cap R=(0)$. Hence $a_{1} \notin A$, so $a_{1} f_{1} \in A$ implies that $f_{1} \in \mathscr{Z}(A)$. Similarly, to show $f_{n} \in \mathscr{Z}(A)$, we wish to show $a_{n} f_{1} \cdots f_{n-1} \notin A$. Consider

Received by the editors September 16, 1971.

AMS 1970 subject classifications. Primary 13E05, 13 F20.

Key words and phrases. Noetherian ring, zero-divisor ring, maximal N-prime.

${ }^{1}$ The authors were supported by National Science Foundation grants GP-29326 and GP-29104.

(c) American Mathematical Society 1972 
the residue class ring $R /\left(a_{1}, \cdots, a_{n-1}\right)=R^{\prime}$. The image of the ideal $A$ in $R^{\prime}[X]$ is generated by the elements $a_{n}^{\prime} f_{1}^{\prime} \cdots f_{n}^{\prime}, a_{n+1}^{\prime} f_{1}^{\prime} \cdots f_{n+1}^{\prime}, \cdots$, where "' " denotes image in $R^{\prime}[X]$. It will suffice to show that

$$
a_{n}^{\prime} f_{1}^{\prime} \cdots f_{n-1}^{\prime} \notin\left(a_{n}^{\prime} f_{1}^{\prime} \cdots f_{n}^{\prime}, a_{n+1}^{\prime} f_{1}^{\prime} \cdots f_{n+1}^{\prime}, \cdots\right) \text {; }
$$

and since $f_{1}^{\prime} \cdots f_{n-1}^{\prime}$ is a monic polynomial in $R^{\prime}[X]$, this is equivalent to showing that $a_{n}^{\prime} \notin\left(a_{n}^{\prime} f_{n}^{\prime}, a_{n+1}^{\prime} f_{n}^{\prime} f_{n+1}^{\prime}, \cdots\right) \subset\left(f_{n}^{\prime}\right)$. Since $f_{n}^{\prime}$ is a monic polynomial of positive degree in $R^{\prime}[X]$, we have $\left(f_{n}^{\prime}\right) \cap R^{\prime}=(0)$. Thus $a_{n}^{\prime} \notin\left(a_{n}^{\prime} f_{n}^{\prime}, a_{n+1}^{\prime} f_{n}^{\prime} f_{n+1}^{\prime}, \cdots\right)$; hence we have proved $f_{n} \in \mathscr{Z}(A)$.

Consider now $f_{i}$ and $f_{j}$ for $i \neq j$. Clearly no prime ideal of $R[X]$ contains both $f_{i}$ and $f_{j}$. Since each $f_{i}$ is in $\mathscr{Z}(A)$ and hence is in some maximal $\mathrm{N}$-prime of $A$, it then follows that $A$ has infinitely many maximal $\mathrm{N}$ primes. Q.E.D. ${ }^{2}$

A Lasker ring is one for which every ideal is a finite intersection of primary ideals. Such rings have been studied by Krull [3], and Evans has observed that every Lasker ring is a ZD-ring. Thus a consequence of the above theorem is that $R$ is noetherian if (and only if) $R[X]$ is Lasker.

We can add one further bit of information on the relationship between the $\mathrm{ZD}$ and noetherian properties.

Proposition. If $R$ is a $Z D$-ring and $R_{P}$ is noetherian for every prime ideal $P$ of $R$, then $R$ is noetherian.

Proof. By [2, Corollary 1.4] it suffices to show that every ideal $A$ of $R$ has only finitely many $B_{\mathrm{w}}$-primes (a $B_{w}$-prime, or weak-Bourbaki prime, of $A$ is a prime ideal $P$ such that $P$ is a minimal prime divisor of $A: x$ for some $x \in R$ ). If $P$ is a $\mathrm{B}_{\mathrm{w}}$-prime of $A$, then $P$ is contained in a maximal $\mathrm{N}$-prime of $A$; and since $R$ is a ZD-ring, $A$ has only a finite number of maximal $\mathrm{N}$-primes, say $Q_{1}, \cdots, Q_{n}$. Moreover, $P$ is a $\mathrm{B}_{\mathrm{w}}$-prime of $A$ in $R$ and $P \subset Q_{i}$ imply $P R_{Q_{i}}$ is a $\mathrm{B}_{\mathrm{w}}$-prime of $A R_{Q_{i}}$ [2, Proposition 1.2]. Since $R_{Q_{i}}$ is noetherian, $A R_{Q_{i}}$ has only a finite number of $\mathrm{B}_{\mathrm{w}}$-primes. Hence $A$ can have only finitely many $\mathrm{B}_{\mathrm{w}}$-primes.

\section{REFERENCES}

1. E. G. Evans, Zero divisors in noetherian-like rings, Trans. Amer. Math. Soc. 155 (1971), 505-512.

2. W. Heinzer and J. Ohm, Locally noetherian commutative rings, Trans. Amer. Math. Soc. 158 (1971), 273-284.

3. W. Krull, Über Laskersche Ringe, Rend. Circ. Mat. Palermo (2) 7 (1958), 155-166. MR 23 \#A1664.

4. D. Underwood, On some uniqueness questions in primary representations of ideals, J. Math. Kyoto Univ. 9 (1969), 69-94. MR 40 \#134.

Department of Mathematics, Purdue University, Lafayette, Indiana 47907

\footnotetext{
${ }^{2}$ We are indebted to the referee for suggesting a judicious choice of the $f_{i}$,s, thus considerably shortening our original proof.
} 